\title{
(2) OPEN ACCESS \\ The association between vital signs and clinical outcomes in emergency department patients of different age categories
}

\author{
Bart GJ Candel 지, ${ }^{1,2}$ Renée Duijzer, ${ }^{2}$ Menno I Gaakeer, ${ }^{3}$ Ewoud ter Avest (1) , ${ }^{4}$ \\ Özcan Sir, ${ }^{5}$ Heleen Lameijer $\odot{ }^{6}{ }^{6}$ Roger Hessels, ${ }^{7}$ Resi Reijnen, ${ }^{8}$ Erik W van Zwet, ${ }^{9}$ \\ Evert de Jonge, ${ }^{10}$ Bas de Groot ${ }^{2}$
}

Handling editor Kirsty Challen

Additional supplemental material is published online only. To view, please visit the journal online (http://dx.doi. org/10.1136/emermed-2020210628)

For numbered affiliations see end of article.

Correspondence to Mr Bart GJ Candel, Emergency Department, Maxima Medical Centre, Veldhoven, The Netherlands; bart.cande|@mmc.nl

Received 8 September 2020 Accepted 7 December 2021

Check for updates

(c) Author(s) (or their employer(s)) 2022. Re-use permitted under CC BY-NC. No commercial re-use. See rights and permissions. Published by BMJ.

To cite: Candel BGJ Duijzer R, Gaakeer Ml, et al. Emerg Med J Epub ahead of print: [please include Day Month Year]. doi:10.1136/

emermed-2020-210628

\section{ABSTRACT}

Background Appropriate interpretation of vital signs is essential for risk stratification in the emergency department (ED) but may change with advancing age. In several guidelines, risk scores such as the Systemic Inflammatory Response Syndrome (SIRS) and Quick Sequential Organ Failure Assessment (qSOFA) scores, commonly used in emergency medicine practice (as well as critical care) specify a single cut-off or threshold for each of the commonly measured vital signs. Although a single cut-off may be convenient, it is unknown whether a single cut-off for vital signs truly exists and if the association between vital signs and in-hospital mortality differs per age-category.

Aims To assess the association between initial vital signs and case-mix adjusted in-hospital mortality in different age categories.

Methods Observational multicentre cohort study using the Netherlands Emergency Department Evaluation Database (NEED) in which consecutive ED patients $\geq 18$ years were included between 1 January 2017 and 12 January 2020. The association between vital signs and case-mix adjusted mortality were assessed in three age categories $(18-65 ; 66-80$; >80 years) using multivariable logistic regression. Vital signs were each divided into five to six categories, for example, systolic blood pressure (SBP) categories ( $\leq 80,81-100,101-120,121-140$, $>140 \mathrm{~mm} \mathrm{Hg}$.

Results We included 101416 patients of whom 2374 (2.3\%) died. Adjusted ORs for mortality increased gradually with decreasing SBP and decreasing peripheral oxygen saturation $\left(\mathrm{SpO}_{2}\right)$. Diastolic blood pressure (DBP), mean arterial pressure (MAP) and heart rate (HR) had quasi-U-shaped associations with mortality. Mortality did not increase for temperatures anywhere in the range between $35.5^{\circ} \mathrm{C}$ and $42.0^{\circ} \mathrm{C}$, with a single cut-off around $35.5^{\circ} \mathrm{C}$ below which mortality increased. Single cut-offs were also found for MAP $<70 \mathrm{~mm} \mathrm{Hg}$ and respiratory rate $>22 / \mathrm{min}$. For all vital signs, older patients had larger increases in absolute mortality compared with younger patients.

Conclusion For SBP, DBP, $\mathrm{SpO}_{2}$ and $\mathrm{HR}$, no single cutoff existed. The impact of changing vital sign categories on prognosis was larger in older patients. Our results have implications for the interpretation of vital signs in existing risk stratification tools and acute care guidelines.
Key messages

What is already known on this subject

- Many risk stratification tools and acute care guidelines specify a single cut-off for vital signs suggesting that above or below this cut-off prognosis changes substantially.

- It is unknown whether relevant single cut-off values for vital signs truly exist.

- In older age, physiological changes may cause differences in the single cut-offs for vital signs and the need for different interpretation.

\section{What this study adds}

- This retrospective study of 101416 emergency department visits from three hospitals showed that mortality and high dependency care unit admission increased gradually or in a Ushaped fashion with changing vital signs. Older patients had larger increases in absolute risks for mortality with changing vital signs.

- Relevant single cut-offs did not exist for systolic blood pressure or oxygen saturation; cut-offs were found for respiratory rate, temperature and mean arterial pressure; however, using these single cut-offs would ignore further increase of risk with more extreme values of these vital signs.

- The use of a single cut-off for each vital sign in risk stratification tools like SIRS and qSOFA and acute care guidelines deserves scrutiny; risk tools should consider using age-adjusted numerical scores.

\section{INTRODUCTION}

Vital signs are used for risk stratification in nearly all acute care guidelines. ${ }^{1-4}$ Many risk stratification tools, such as the Systemic Inflammatory Response Syndrome (SIRS), Quick Sequential Organ Failure Assessment (qSOFA) and CURB-65 scores, ${ }^{2}$ 5-8 specify a single cut-off (or threshold) for each of the commonly measured vital signs, suggesting that one cut-off may discriminate between good and bad prognosis. However, recent studies in patients with traumatic brain injury and sepsis showed that prognosis linearly worsened with decreasing systolic blood pressure (SBP), without an identifiable single threshold. ${ }^{9}{ }^{10}$ Similarly, linear or U-shaped instead 
of dichotomous associations may exist for other vital signs used in ED risk stratification.

In addition, most risk stratification models are applied in all ED patients irrespective of age. However, a recent study showed that risk stratification tools are inappropriate for older ED patients. ${ }^{11} 12$ The association between vital signs and outcomes may change with advancing age due to physiological changes. Arterial walls become stiffer in older patients, heart rate (HR) response to stress is blunted and fever is absent in many older patients with sepsis. ${ }^{13}$

Although a single cut-off for vital signs may be convenient in risk stratification tools, numerical scores based on predicted mortality will possibly better inform physicians and prevent impaired recognition of early deterioration in ED patients. Physiological changes with increasing age may implicate that currently used risk stratification tools should be age-adjusted. This is particularly important given the ageing of the population with an increasing number of older ED patients.

Therefore, the aims of the present study are twofold: First, to assess the associations of vital signs and relevant clinical outcomes (eg, mortality and intensive care unit (ICU)/medium care unit (MCU) admission) and whether a single cut-off or threshold exist for each vital sign, and second, to study whether associations of vital signs and relevant clinical outcomes change with advancing age.

\section{METHODS}

\section{Study design and setting}

This observational multicentre study was conducted in three EDs in the Netherlands, each with approximately 25 000-30 000 ED visits per year. Data from the three sites spanned slightly different times: data from the tertiary care centre included visits between 1 January 2017 and 8 June 2019, and data from the two urban teaching hospitals included visits between 1 January 2019 to 12 January 2020 and 1 January 2017 to 31 December 2019, respectively. The tertiary care centre uses the Manchester Triage System and both urban hospitals use the Dutch Triage Standard. The study was registered in the Netherlands Trials Register: NL8422.

\section{Selection of participants}

All consecutive ED patients $\geq 18$ years were included if one or more of the following vital signs were measured: respiratory rate (RR), peripheral oxygen saturation $\left(\mathrm{SpO}_{2}\right), \mathrm{SBP}$, diastolic blood pressure (DBP), mean arterial blood pressure (MAP), HR and temperature.

\section{Data collection}

Data were collected from the Netherlands Emergency department Evaluation Database (NEED), the Dutch quality registry for EDs (www.stichting-need.nl), a record containing clinical data from all ED visits from the participating hospitals. Online supplemental file 1 provides a detailed description of the data collected in the NEED. Twice a year all clinical data from the participating hospitals is downloaded into the record. Onset of participation in the NEED differs per hospital. For the current study, data were available from three of the nine participating hospitals. The NEED stores data using the web-based application Project Manager Internet Server (ProMISe, Leiden, the Netherlands, https://www.msbi.nl/promise/promise.aspx). Privacy sensitive data are encrypted by a trusted third party (ZorgTTP, Houten, the Netherlands, www.zorgttp.nl) with Trusted Reversible Encryption Service.
The vital signs measured at the beginning of ED presentation, before ED treatment, were recorded in the NEED. Only one set of vital signs was recorded per patient. If patients arrived by own transport, $\mathrm{HR}, \mathrm{SpO}_{2}$ and temperature were measured in a triage room by a nurse mostly in seated, sometimes supine, position. These vital signs were entered manually in the hospital information system. Once moved to a patient room, the other vital signs were measured by a nurse with the MP52 IntelliVue, Intellivue MP30, Intellivue MX400 or MX500 monitor (Philips, Eindhoven, the Netherlands). Multipatient reusable latex-free NIBP Comfort Care Cuff from Philips are used with cuff a small cuff $(27-35 \mathrm{~cm})$, medium cuff $(35-45 \mathrm{~cm})$, large cuff $(42-54$ $\mathrm{cm})$ and extra-large cuff $(44-56 \mathrm{~cm})$, depending on the size of the patient. Vital signs registered in the patient room were automatically transferred in the hospital information system, except for temperature, which was entered manually. Temperature was measured with a Genius 3 Tympanic Thermometer. If patients arrived by ambulance, vital signs were measured in supine position in the patient room as described. In the tertiary care centre, vital signs were automatically measured every $2.5-15 \mathrm{~min}$ and transferred to the electronic patient file with PDMS software (Chipsoft, the Netherlands), depending on the personalised setting for a specific patient. Median values of vital signs were registered for the first $10 \mathrm{~min}$ of measuring.

\section{Measurements}

Before we could perform statistical analysis, several variables had to be synchronised among hospitals. Beforehand, we could not assume that the association between vital signs and outcomes were linear. Therefore, vital signs were categorised into ranges based on expected distribution and commonly used reference intervals: RR (not measured, 0-9, 10-19, 20-29, $\geq 30 / \mathrm{min}$ ), $\mathrm{SpO}_{2}$ (0\%-80\%, 81\%-85\%, 86\%-90\%, 91\%-95\%, 96\%-100\%), SBP (0-80, 81-100, 101-120, 121-140, >140 mm Hg), DBP (0-60, 61-80, 81-100, 101-120, >120 mm Hg), MAP (0-60, 61-80, 81-100, 101-120, >120 mm Hg), HR (0-50, 51-75, 76-100, 101-125, >125beats/min.), temperature (not measured, 0-30, 31-34, 35-37, 38-39, $\geq 40^{\circ} \mathrm{C}$ ).

Correspondent to literature, ${ }^{14} \mathrm{RR}$ and temperature were often not registered. To limit the number of missings in the analyses, we included a category 'not registered'. In EDs in the Netherlands, vital signs are not registered in all patients. For example, vital signs are often not registered in patients with an ankle distortion or a single fracture, because they are at low risk of adverse events and often discharged, and RR is often only registered if patients are considered critically ill by the nurse. Therefore, the fact that vital signs are registered may provide relevant information for risk stratification. Online supplemental file 2 describes how the variables triage levels, chief complaints (the top 10 chief complaints was used) and treating specialties were modified before they could be used as potential confounders in the statistical analyses.

Patients were stratified into three age categories: 18-65, $66-80$ and $>80$ years. These age categories were chosen based on the mean SBP in the general population in the Netherlands according to the National Institute for Public Health and the Environment (RIVM, www.rivm.nl). The mean SBP increases substantially above 65 years.

\section{Outcome}

The primary outcome was whether there was a vital sign category that could be used as a cut-off to predict the outcomes of 
in-hospital mortality and ICU or MCU admission, which also included the Coronary Care Unit.

\section{Sample size estimation}

Approximately 5-10 events per variable are needed to prevent overfitting in association studies. ${ }^{15}$ The NEED contained 148828 ED visits of patients $\geq 18$ years. We estimated that in $\sim 60 \%$ of the ED visits vital signs were registered resulting in $\sim 90000$ ED visits which could be used for the analyses. In-hospital mortality was estimated to be $\sim 3 \%$. Included patients were stratified in three age categories, yielding $\sim 90$ $000 / 3=30000$ patients per age category. Thus, we expected to have $\sim 0.03 \times 30,000=900$ events per group to be able to adjust for the 37 potential confounders (see main statistical analysis) in the analyses with in-hospital mortality as outcome, which is appropriate to prevent overfitting.

\section{Statistical analyses}

Patient characteristics were described as mean (SD) for normally distributed data, and median (IQR) for skewed data. Categorical data were presented as number $(\mathrm{N},(\%))$.

For all analyses, one category per vital sign was used as reference category (ie, reference category for HR was $50-75$ beats/ min). The number of measured vital signs outside the reference range were calculated.

We used multivariable logistic regression analyses for the association between vital sign categories and the primary and secondary outcomes. The following potential confounders were entered in the models for which then backward stepwise elimination was performed: age, sex, triage level, top 10 chief complaints, hospital, treating specialty, disposition (ward, ICU/ $\mathrm{MCU}), \mathrm{SpO}_{2}$, temperature (including category 'not registered'), Glasgow Coma Scale (GCS) (not assessed, assessed and 15 points, assessed and $<15$ points), lab tests $(0=$ no lab tests performed, $1=$ any lab test performed $)$, blood gas analyses $(0=$ not tested, $1=$ tested), number of consultations in the $\operatorname{ED}(0,1,2$ or $\geq 3$ consultations) and performed radiological test $(0=$ none, $1=$ any test). Other vital signs were not entered in the models because of potential multicollinearity among vital signs. The number of consultations, lab tests and radiological tests were used as measures of comorbidities/complexity, as has been suggested previously. ${ }^{16} 17$ Triage level, GCS, blood gas analyses and ICU/ MCU admission were used as disease severity measures. ${ }^{11} \mathrm{ICU} /$ MCU admission is an outcome and also a proxy of disease severity not captured in the initial vital signs. For example, a patient with an initial SBP of $90 \mathrm{~mm} \mathrm{Hg}$ who responds to fluid resuscitation can be admitted to a normal ward and has a different risk for in-hospital mortality compared with a patient who also has an initial SBP of $90 \mathrm{~mm} \mathrm{Hg}$ does not respond to fluid resuscitation and consequently needs ICU admission. In other words, the variable ICU/MCU admission also reflects the response to treatment. For the secondary outcome, the potential confounder disposition was replaced by the amount of fluid administration as a proxy of disease severity. Multicollinearity was assumed not to be a problem if variation inflation factors were below three. The associations between vital sign categories and case-mix adjusted outcomes were assessed in two ways to give better insight in differences between age categories, as the relative risk (AORs) for the outcome may be similar among age categories, while the absolute risk may be different due to different baseline risk at the reference values. We used adjusted odds ratios (AORs) with 95\% CI as relative risk measure and predicted (case mix-adjusted) mortality as absolute risk measure.
In the figures, the AORs of in-hospital mortality were plotted as a function of vital sign categories for the pooled data and for all age categories. If we observed a sudden rise or decline in AORs by changing vital sign categories, we performed minimal $\mathrm{p}$ value analyses to assess a more precise value for the possible threshold. In these analyses, we changed the vital sign into a binary value (below or above a cut-off value) in the multivariable logistic regression as described above. We repeated the analyses with all possible cut-off values in steps of $0.5^{\circ} \mathrm{C}$ (temperature), 1/ min. (RR), $5.0 \mathrm{~mm} \mathrm{Hg}$ (SBP, DBP, MAP), 5.0 beats/min (HR) or $5.0 \%\left(\mathrm{SpO}_{2}\right)$. The cut-off value with the lowest $\mathrm{p}$ value for the AOR was considered as threshold. In this way, a threshold was determined in approximately continuous data of the vital sign. Thresholds with minimal $\mathrm{p}$ value analyses were not determined if vital signs had no clear single cut-off.

$\mathrm{P}<0.05$ was considered as statistically significant. Data were analysed using SPSS (V.25.0, IBM, New York, USA).

\section{Subgroup and sensitivity analyses}

We performed subgroup analyses for suspected infection and trauma patients to assess whether aetiology affected the primary associations of interest. See online supplemental files 3 and 4 for case definitions. A sensitivity analyses was performed to assess whether hospital transfers may have influenced our results, as we did not follow-up on these patients. The main analyses were repeated in which patients transferred to other hospitals were considered as deceased.

\section{Patient and public involvement}

No patients were involved.

\section{RESULTS}

\section{Patient inclusion and characteristics}

Figure 1 shows that of the 147728 ED visits of patients $\geq 18$ years, 101416 patients were included in whom vital signs were measured.

Table 1 shows patient characteristics of the total cohort and per age-category. The mean age of included patients was 59.6 years (19.6). Table 1 also shows the percentage of patients in whom vital signs fell outside the reference ranges which are

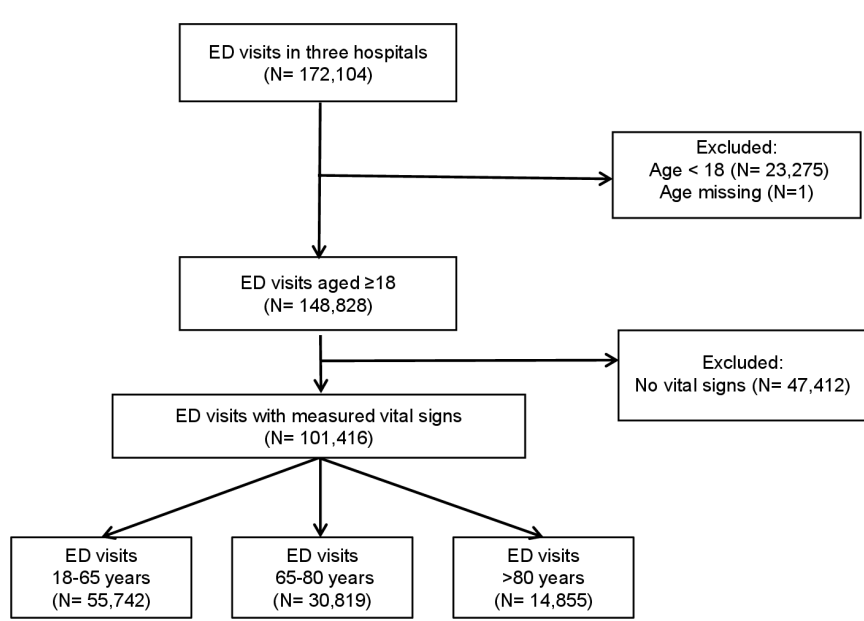

Figure 1 Patient inclusion and flow through the study. Observational multicentre cohort study using the NEED. Three different age categories were included in which vital signs were measured. ED, emergency department; NEED, Netherlands Emergency Department Evaluation Database. 


\section{Original research}

Table 1 Patient characteristics for the total cohort and different age categories

\begin{tabular}{|c|c|c|c|c|}
\hline & Total cohort, N=101 $416(100 \%)$ & $18-65$ years, $\mathrm{N}=55742(55 \%)$ & $66-80$ years, $\mathrm{N}=30819(30 \%)$ & $>80$ years, $\mathrm{N}=14855(15 \%)$ \\
\hline \multicolumn{5}{|l|}{ Demographics N, (\%) } \\
\hline Sex, female & $50333(49.6)$ & $28226(50.7)$ & $13752(44.6)$ & $8315(56.0)$ \\
\hline Age, mean (SD) & $59.6(19.6)$ & $45.3(14.1)$ & $72.9(4.2)$ & $85.8(3.9)$ \\
\hline \multicolumn{5}{|l|}{ Hospital setting N, (\%) } \\
\hline LUMC & $28361(28.0)$ & $17980(32.3)$ & $7536(24.5)$ & $2845(19.2)$ \\
\hline $\mathrm{MCL}$ & $53378(52.6)$ & $27048(48.5)$ & $17698(57.4)$ & $8632(58.1)$ \\
\hline CZE & $19677(19.4)$ & $10714(19.2)$ & $5585(18.1)$ & $3378(22.7)$ \\
\hline \multicolumn{5}{|l|}{ Treating specialty N, (\%) } \\
\hline Emergency medicine & $17656(17.4)$ & $11062(19.8)$ & $4274(13.9)$ & $2320(15.6)$ \\
\hline Surgical & $19434(19.2)$ & $11902(21.4)$ & $5076(16.5)$ & $2456(16.5)$ \\
\hline Medical & $62262(61.4)$ & $31096(55.8)$ & $21157(68.6)$ & $10009(67.4)$ \\
\hline \multicolumn{5}{|c|}{ Top-10 chief complaints $\mathrm{N},(\%)$} \\
\hline Feeling unwell & $20414(20.1)$ & $9192(16.5)$ & $7392(24.4)$ & $3830(26.3)$ \\
\hline Abdominal pain & $14185(14.0)$ & $10136(18.2)$ & $3035(10.0)$ & $1014(7.0)$ \\
\hline Dyspnoea & 11937 (11.8) & 4975 (8.9) & $4673(15.4)$ & $2289(15.7)$ \\
\hline Chest pain & $11067(10.9)$ & $6327(11.4)$ & $3523(11.6)$ & $1217(8.4)$ \\
\hline Extremity problems & $8106(8.0)$ & $3736(6.7)$ & $2422(8.0)$ & $1948(13.4)$ \\
\hline Collapse & $3868(3.8)$ & $1722(3.1)$ & $1433(4.7)$ & $713(4.9)$ \\
\hline Trauma & $3778(3.7)$ & $2263(4.1)$ & $876(2.9)$ & $639(4.4)$ \\
\hline Palpitations & $3376(3.3)$ & $1665(3.0)$ & $1370(4.5)$ & $341(2.3)$ \\
\hline Wounds & $2256(2.2)$ & $1442(2.6)$ & $601(2.0)$ & $213(1.5)$ \\
\hline Headache & $2144(2.1)$ & $1465(2.6)$ & $475(1.6)$ & $204(1.4)$ \\
\hline Miscellaneous & $18311(18.2)$ & $11437(22.9)$ & $4465(14.9)$ & $2147(14.7)$ \\
\hline \multicolumn{5}{|c|}{ Vital signs, Mean (SD)(N) $\{\%$ outside of reference category* $\}$} \\
\hline $\mathrm{RR}(/ \mathrm{min})$ & $18(6)(68662)\{23.4\}$ & $17(6)(34923)\{18.3\}$ & $19(6)(22545)\{27.1\}$ & $19(6)(11194)\{32.1\}$ \\
\hline $\mathrm{SPO}_{2}(\%)$ & $97(6)(91785)\{14.2\}$ & $99(3)(49,628)\{8.5\}$ & $96(9)(28.374)\{18.1\}$ & $96(4)(13,783)\{23.6\}$ \\
\hline $\mathrm{SBP}(\mathrm{mm} \mathrm{Hg})$ & $133(34)(89531)\{79.1\}$ & $128(28)(47,444)\{76.3\}$ & $\begin{array}{l}137(40)(28,189) \\
\{81.7\}\end{array}$ & $141(34)(13898)\{83.3\}$ \\
\hline $\mathrm{DBP}(\mathrm{mm} \mathrm{Hg})$ & $83(17)(89475)\{60.7\}$ & $84(16)(47424)\{62.5\}$ & $81(18)(28169)\{58.7\}$ & $80(19)(13882)\{58.3\}$ \\
\hline MAP (mm Hg) & $99(19)(89512)\{50.9\}$ & $98(18)(47,438)\{47.9\}$ & $100(21)(28181)\{53.8\}$ & $100(21)(12407)\{54.7\}$ \\
\hline HR (beats/min) & $86(52)(84776)\{23.4\}$ & $87(69)(44,611)\{18.3\}$ & $86(22)(26978)\{27.1\}$ & $83(21)(13187)\{32.1\}$ \\
\hline Temperature $\left({ }^{\circ} \mathrm{C}\right)$ & $37.0(2.0)(82364)\{42.1\}$ & $37.0(0.8)(45073)\{39.7\}$ & $37.0(3.3)(25349)\{44.6\}$ & $36.9(0.9)(11942)\{45.8\}$ \\
\hline \multicolumn{5}{|c|}{ Proxies of disease severity $N,(\%)$} \\
\hline \multicolumn{5}{|l|}{ Triage level } \\
\hline Blue and green & $22414(22.1)$ & $12952(23.2)$ & $6222(20.2)$ & $3240(21.8)$ \\
\hline Yellow & $43804(43.2)$ & $24511(44)$ & $13021(42.2)$ & $6272(42.2)$ \\
\hline Orange & $28176(27.8)$ & $14750(26.5)$ & $9198(29.8)$ & $4228(28.5)$ \\
\hline Red & $5339(5.3)$ & $2590(4.6)$ & $1896(6.2)$ & $853(5.7)$ \\
\hline \multicolumn{5}{|l|}{ GCS } \\
\hline Not assessed & $91143(89.9)$ & $51021(91.5)$ & $27288(88.5)$ & $12834(86.4)$ \\
\hline 15 & $9041(8.9)$ & $4154(7.5)$ & $3170(10.3)$ & $1717(11.6)$ \\
\hline$<15$ & $1232(1.2)$ & $567(1.0)$ & $361(1.2)$ & $304(2)$ \\
\hline \multicolumn{5}{|l|}{ Fluid administration } \\
\hline None & $82182(81)$ & $45626(81.9)$ & $24623(79.9)$ & $11933(80.3)$ \\
\hline $0-500 \mathrm{~mL}$ & $9164(9.0)$ & $4734(8.5)$ & $2909(9.4)$ & $1521(10.2)$ \\
\hline$>500 \mathrm{~mL}$ & $10070(9.9)$ & $5382(9.7)$ & $3287(10.7)$ & $1401(9.4)$ \\
\hline Blood gas analyses & $21750(21.4)$ & $9874(17.7)$ & $7924(25.7)$ & $3952(26.6)$ \\
\hline \multicolumn{5}{|c|}{ Proxies of comorbidities/complexity N, (\%) } \\
\hline \multicolumn{5}{|l|}{ Number of consultations } \\
\hline None & $34657(34.2)$ & $20575(36.9)$ & $9671(31.4)$ & $4411(29.7)$ \\
\hline 1 & $57810(57.0)$ & $30107(54)$ & $18633(60.5)$ & $9070(61.1)$ \\
\hline 2 & $6369(6.3)$ & $3193(5.7)$ & $2012(6.5)$ & $1164(7.8)$ \\
\hline$>2$ & $829(0.8)$ & $417(0.7)$ & $257(0.8)$ & $155(1.0)$ \\
\hline$\geq 1$ lab test & $84434(83.3)$ & $44232(79.4)$ & $27144(88.1)$ & $13058(87.9)$ \\
\hline Radiological test $†$ & $60739(59.9)$ & $29741(53.4)$ & $19908(64.6)$ & $11090(74.7)$ \\
\hline
\end{tabular}

*Used reference categories were: SBP 100-140 mm Hg, DBP 60-90 mm Hg, MAP 70-100 mm Hg, HR 60-80 beats/min, RR 12-20/min. SpO $2995 \%$, temperature 36.5-37.5C.

tIf one or more of the following radiological tests were performed: ultrasound, radiography and CT.

CZE, Catharina Hospital Eindhoven; DBP, diastolic blood pressure; GCS, Glasgow Coma Scale; HR, heart rate; LUMC, Leiden University Medical Center; MAP, mean arterial pressure; MCL, Medical Centre Leeuwarden; N, number; RR, respiratory rate; SBP, systolic blood pressure; SPO2, peripheral oxygen saturation. 
Table 2 Adjusted Odds Ratio's for the association between vital signs and in-hospital mortality by different age groups

\begin{tabular}{|c|c|c|c|c|}
\hline & Total cohort & $18-65$ years & $66-80$ years & $>80$ years \\
\hline \multicolumn{5}{|c|}{ Systolic blood pressure $(\mathrm{mm} \mathrm{Hg})$} \\
\hline$>140^{*}$ & 1.00 & & & \\
\hline $121-140$ & $1.35(1.18-1.54)$ & $1.68(1.22-2.33)$ & $1.28(1.04-1.57)$ & $1.39(1.12-1.72)$ \\
\hline $101-120$ & $1.91(1.67-2.19)$ & $2.50(1.81-3.45)$ & $1.75(1.42-2.16)$ & $1.97(1.57-2.46)$ \\
\hline $81-100$ & $2.62(2.22-3.09)$ & $2.82(1.92-4.13)$ & $2.83(2.21-3.61)$ & $2.44(1.84-3.25)$ \\
\hline $0-80$ & $4.07(3.31-5.01)$ & $6.38(4.20-9.70)$ & $3.33(2.41-4.60)$ & $4.17(2.87-6.05)$ \\
\hline \multicolumn{5}{|c|}{ Diastolic blood pressure $(\mathrm{mm} \mathrm{Hg})$} \\
\hline$>120$ & $1.73(1.37-2.19)$ & $1,82(1.08-3.07)$ & $2.25(1.57-3.20)$ & $1.26(0.84-1.88)$ \\
\hline $101-120$ & $1.15(0.96-1.37)$ & $1.48(1.04-2.10)$ & $1,24(0.95-1.63)$ & $0.88(0.65-1.20)$ \\
\hline $81-100^{*}$ & 1.00 & & & \\
\hline $61-80$ & $1.23(1.09-1.39)$ & $1.62(1.24-2.11)$ & $1,30(1.08-1.56)$ & $0.99(0.82-1.21)$ \\
\hline $0-60$ & $2.12(1.84-2.44)$ & $3.42(2.47-4.73)$ & $2,32(1.87-2.88)$ & $1.59(1.27-2.00)$ \\
\hline \multicolumn{5}{|c|}{ Mean Arterial blood pressure $(\mathrm{mm} \mathrm{Hg})$} \\
\hline \multicolumn{5}{|c|}{$>120^{*}$} \\
\hline $101-120$ & $0.88(0.75-1.03)$ & $1.01(0.68-1.50)$ & $0.81(0.64-1.02)$ & $0.95(0.74-1.23)$ \\
\hline $81-100$ & $1.06(0.90-1.23)$ & $1.56(1.07-2.27)$ & $0.88(0.70-1.12)$ & $1.11(0.86-1.42)$ \\
\hline $61-80$ & $1.73(1.47-2.05)$ & $2.02(1.33-3.07)$ & $1.72(1.33-2.21)$ & $1.75(1.32-2.30)$ \\
\hline $0-60$ & $3.27(2.60-4.10)$ & $5.72(3.48-9.42)$ & $2.86(2.01-4.09)$ & $3.17(2.17-4.64)$ \\
\hline \multicolumn{5}{|c|}{ Peripheral oxygen saturation (\%) } \\
\hline $96-100^{*}$ & 1.00 & & & \\
\hline $91-95$ & $1.46(1.31-1.62)$ & $1.68(1.30-2.18)$ & $1.30(1.10-1.53)$ & $1.48(1.25-1.77)$ \\
\hline $86-90$ & $2.18(1.83-2.61)$ & $3.28(2.17-4.94)$ & $1.84(1.41-2.39)$ & $2.22(1.66-2.97)$ \\
\hline $81-85$ & $3.58(2.80-4.58)$ & $3.70(2.08-6.57)$ & $2.66(1.81-3.92)$ & $4.92(3.32-7.29)$ \\
\hline $0-80$ & $6.28(5.05-7.82)$ & $11.1(7.40-16.6)$ & $4.50(3.19-6.34)$ & $4.99(3.30-7.56)$ \\
\hline \multicolumn{5}{|c|}{ Respiratory rate (/min) } \\
\hline Not registered* & 1.00 & & & \\
\hline $0-9$ & $0.99(0.49-2.01)$ & $0.79(0.24-2.69)$ & $1.54(0.58-4.07)$ & $0.58(0.08-4.46)$ \\
\hline $10-19$ & $1.10(0.92-1.30)$ & $0.82(0.60-1.13)$ & $1.15(0.87-1.51)$ & $1.41(1.02-1.94)$ \\
\hline $20-29$ & $1.83(1.53-2.18)$ & $1.47(1.04-2.06)$ & $1.89(1.43-2.50)$ & $2.28(1.64-3.15)$ \\
\hline$>30$ & $2.89(2.32-3.60)$ & $2.57(1.62-4.06)$ & $3.23(2.31-4.53)$ & $3.17(2.15-4.68)$ \\
\hline \multicolumn{5}{|c|}{ Heart rate (beats/min) } \\
\hline$>125$ & $2.98(2.44-3.64)$ & $4.17(2.77-6.29)$ & $2.47(1.79-3.41)$ & $2.76(1.95-3.90)$ \\
\hline $101-125$ & $2.50(2.16-2.91)$ & $2.61(1.85-3.69)$ & $2.71(2.16-3.40)$ & $2.22(1.72-2.86)$ \\
\hline $76-100$ & $1.43(1.25-1.63)$ & $1.34(0.97-1.85)$ & $1.48(1.21-1.82)$ & $1.48(1.21-1.81)$ \\
\hline \multicolumn{5}{|l|}{$51-75^{*}$} \\
\hline $0-50$ & $1.61(1.19-2.17)$ & $2.04(1.02-4.08)$ & $1.67(1.04-2.69)$ & $1.54(0.96-2.45)$ \\
\hline \multicolumn{5}{|l|}{ Temperature $\left({ }^{\circ} \mathrm{C}\right)$} \\
\hline \multicolumn{5}{|l|}{ Not registered* } \\
\hline$\geq 40$ & $0.49(0.28-0.86)$ & $0.40(0.12-1.33)$ & $0.43(0.17-1.10)$ & $0.65(0.26-1.60)$ \\
\hline $38-39$ & $0.68(0.57-0.80)$ & $0.69(0.47-1.00)$ & $0.74(0.57-0.95)$ & $0.57(0.42-0.78)$ \\
\hline $35-37$ & $0.89(0.78-1.00)$ & $0.91(0.69-1.18)$ & $0.81(0.67-0.98)$ & $0.97(0.78-1.20)$ \\
\hline $31-34$ & $2.74(1.96-3.82)$ & $4.11(2.25-7.50)$ & $2.10(1.23-3.58)$ & $2.77(1.52-5.04)$ \\
\hline $0-30$ & $1.54(0.52-4.54)$ & $1.17(0.15-9.34)$ & $0.60(0.06-5.74)$ & $4.04(0.64-25.6)$ \\
\hline
\end{tabular}

The following potential confounders were entered in the model through backward stepwise regression: age, gender, top 10 chief complaints, hospital, treating specialty, $\mathrm{SpO}_{2}$, temperature, proxies of disease severity (triage level, Glasgow Coma Scale, blood gas analyses, intensive care unit admission) and proxies of disease comorbidities/complexity (number of consultations, lab tests, performed radiological tests).

${ }^{*}$ Reference category in the regression analyses.

AOR, adjusted odds ratio.

normally used in adults. For most values, the rate of abnormal vital signs increased with advancing age.

\section{The association between vital signs and clinical outcomes}

The AORs for predicted mortality (table 2) increased gradually with worsening values of SBP and $\mathrm{SpO}_{2}$, while DBP, MAP and HR had a quasi-U-shaped association with mortality (figures 2 and 3). There was no clear cut-off point between categories of SBP, DBP, $\mathrm{SpO}_{2}$ and HR and AORs for mortality. The AOR for RR gradually increased between 10 and $19 \mathrm{~mm}$ (figure 3), with a substantial increase for mortality at $22 / \mathrm{min}$. The AOR of mortality showed a substantial increase for temperature below a threshold of $35.5^{\circ} \mathrm{C}$; however, temperatures above this were not associated with mortality. For MAP, a threshold at $<70 \mathrm{~mm}$ $\mathrm{Hg}$ was found for mortality. Although single cut-offs for MAP, $\mathrm{RR}$ and temperature exist, using these cut-offs would ignore further increase of risk with more extreme values of these vital signs. 

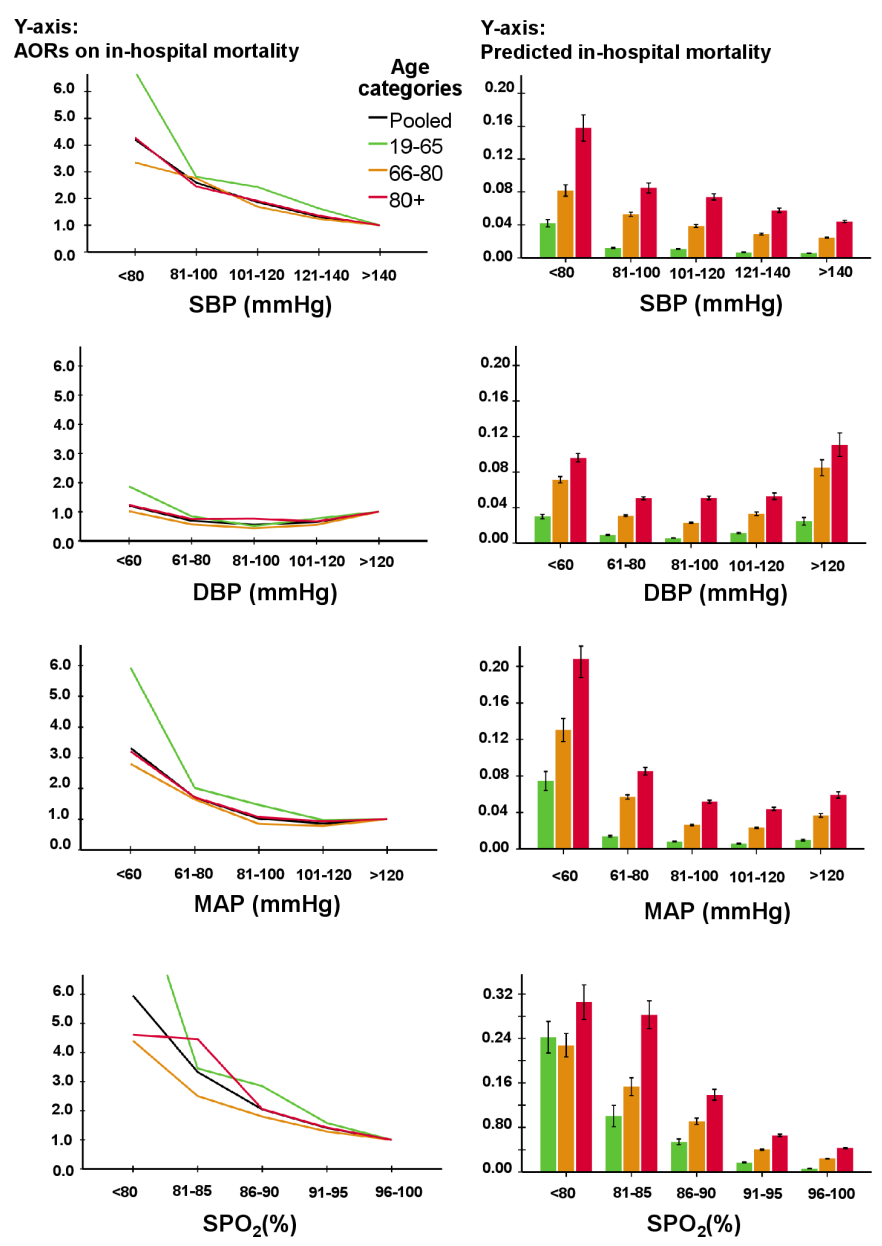

Figure 2 AORs for SBP, DBP, MAP and $\mathrm{SpO}_{2}$ for in-hospital mortality (left side) and predicted in-hospital mortality (right side) as a function of vital signs in different age categories. Note that the black line is the pooled data of all three age categories together. The following potential confounders were entered in the model for in-hospital mortality through backward stepwise regression: age, gender, triage level, top 10 chief complaints, hospital, treating specialty, disposition, $\mathrm{SpO}_{2}$ (except for association of $\mathrm{SpO}_{2}$ ), temperature (except for association of temperature), Glasgow Coma Scale, lab tests, number of consultations in the emergency department and performed radiological test. AORs, adjusted odds ratios; DBP, diastolic blood pressure; MAP, mean arterial pressure; $\mathrm{SBP}$, systolic blood pressure; $\mathrm{SpO}_{2}$, peripheral oxygen saturation.

Older patients had larger increases in case-mix adjusted predicted mortality compared with younger people with changing vital sign categories (right panels in figures 2 and 3). In contrast, AORs for mortality decreased or increased to a similar extent with changing vital sign categories. Only patients aged 18-65 years with an $\mathrm{SpO}_{2}<80 \%$ had higher odds for mortality (AOR $11.09 ; 7.40-16.62)$ compared with patients aged $66-80$ years (AOR $4.50 ; 3.19-6.34$ ) and $>80$ years (AOR $4.99 ; 3.30-7.56$ ).

For ICU/MCU admission (table 3, online supplemental files 5 and 6), SBP had a relevant cut-off in the category of $<80 \mathrm{~mm}$ $\mathrm{Hg}$ and was calculated at $70 \mathrm{~mm} \mathrm{Hg}$ with minimal $\mathrm{p}$ value analysis. For MAP, a threshold of $<60 \mathrm{~mm} \mathrm{Hg}$ existed for admission to the ICU/MCU. When RR was registered, AOR for ICU admission was at least doubled compared with when it was not recorded, but no other cut-off was seen. Temperature $<31^{\circ} \mathrm{C}$ had a substantially higher likelihood for ICU admission.
Y-axis:

AORs on in-hospital mortality

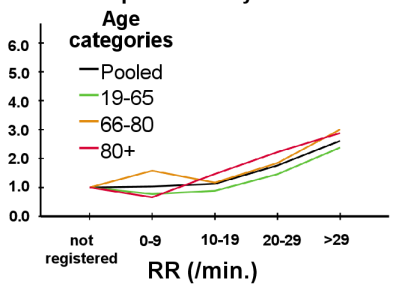

Y-axis:

Predicted in-hospital mortality
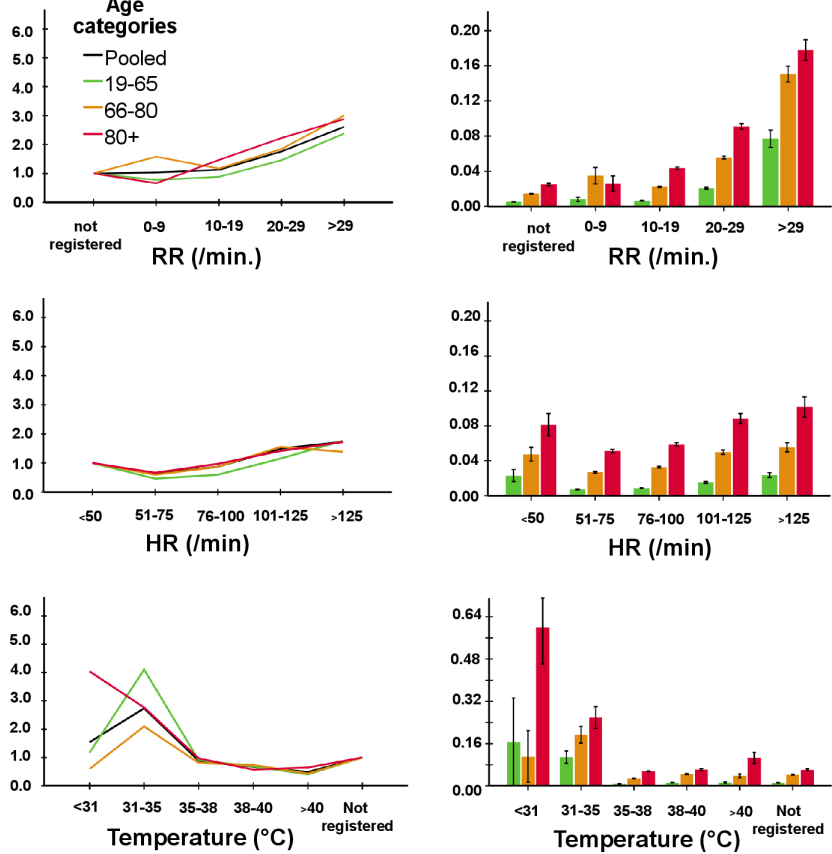

Figure 3 AORs for RR, HR and temperature for in-hospital mortality (left side) and predicted in-hospital mortality (right side) as a function of vital signs in different age categories. Note that the black line is the pooled data of all three age categories together. The following potential confounders were entered in the model for in-hospital mortality through backward stepwise regression: age, gender, triage level, top 10 chief complaints, hospital, treating specialty, disposition, $\mathrm{SpO}_{2}$, temperature, Glasgow coma scale, lab tests, number of consultations in the emergency department and performed radiological test. AORs, adjusted odds ratios; $H R$, heart rate; $R R$, respiratory rate.

Younger patients had larger increases in predicted ICU/MCU admission compared with older patients with increasing DBP, $\mathrm{RR}, \mathrm{HR}$ and decreasing $\mathrm{SpO}_{2}$ categories (online supplemental files 5 and 6). In contrast, AORs changed to a similar extent among all age categories for changing vital signs.

\section{Subgroup and sensitivity analyses}

The AORs for mortality increased or decreased to the same extent in the subgroups with suspected infection and trauma patients, and in the sensitivity analyses, regardless of the patient's age category (online supplemental files 3, 4 and 7).

\section{DISCUSSION}

The present study has two main findings. First, in-hospital mortality increased gradually with decreasing SBP and $\mathrm{SpO}_{2}$, without the existence of a single threshold for these vital signs. For DBP, MAP and HR, we found quasi-U-shaped associations with in-hospital mortality. A single cut-off existed for MAP, RR and temperature; however, using these single cut-offs would ignore further increase of risk with more extreme values of these vital signs.

Second, absolute mortality increased substantially more in older compared with younger patients with changing vital sign categories.

The use of a single threshold for SBP in many acute care guidelines and risk stratification tools deserves scrutiny. ${ }^{6-8} 1819$ The linear association between SBP and mortality in an unselected ED 
Table 3 AORs for the association between vital signs and ICU/MCU admission by different age groups

\begin{tabular}{|c|c|c|c|c|}
\hline & Total cohort & $18-65$ years & $66-80$ years & $>80$ years \\
\hline \multicolumn{5}{|c|}{ Systolic blood pressure (mm Hg) } \\
\hline$>140^{*}$ & 1.00 & & & \\
\hline $121-140$ & $0.89(0.82-0.97)$ & $0.90(0.79-1.03)$ & $0.95(0.83-1.09)$ & $0.94(0.75-1.17)$ \\
\hline $101-120$ & $0.98(0.89-1.08)$ & $1.01(0.88-1.17)$ & $0.95(0.81-1.12)$ & $1.14(0.88-1.46)$ \\
\hline $81-100$ & $0.97(0.84-1.11)$ & $0.97(0.79-1.18)$ & $0.93(0.74-1.16)$ & $1.21(0.86-1.72)$ \\
\hline $0-80$ & $1.94(1.64-2.30)$ & $2.11(1.63-2.72)$ & $1.98(1.50-2.60)$ & $1.88(1.21-2.94)$ \\
\hline \multicolumn{5}{|c|}{ Diastolic blood pressure $(\mathrm{mm} \mathrm{Hg})$} \\
\hline$>120$ & $2.18(1.85-2.58)$ & $2.34(1.83-3.00)$ & $2.31(1.76-3.03)$ & $1.54(0.98-2.40)$ \\
\hline $101-120$ & $1.34(1.21-1.48)$ & $1.30(1.12-1.51)$ & $1.36(1.15-1.61)$ & $1.34(1.01-1.77)$ \\
\hline $81-100^{*}$ & 1.00 & & & \\
\hline $61-80$ & $0.93(0.86-1.00)$ & $0.99(0.88-1.12)$ & $0.82(0.72-0.93)$ & $1.00(0.81-1.22)$ \\
\hline $0-60$ & $1.32(1.18-1.49)$ & $1.71(1.41-2.07)$ & $1.32(1.10-1.58)$ & $1.04(0.78-1.37)$ \\
\hline \multicolumn{5}{|c|}{ Mean arterial blood pressure $(\mathrm{mm} \mathrm{Hg})$} \\
\hline$>120^{*}$ & 1.00 & & & \\
\hline $101-120$ & $0.74(0.67-0.82)$ & $0.69(0.60-0.81)$ & $0.81(0.70-0.95)$ & $0.73(0.56-0.93)$ \\
\hline $81-100$ & $0.64(0.57-0.70)$ & $0.65(0.56-0.76)$ & $0.62(0.52-0.73)$ & $0.72(0.56-0.93)$ \\
\hline $61-80$ & $0.76(0.67-0.86)$ & $0.78(0.64-0.95)$ & $0.80(0.66-0.98)$ & $0.73(0.53-1.00)$ \\
\hline $0-60$ & $1.70(1.40-2.06)$ & $2.18(1.61-2.96)$ & $1.89(1.38-2.58)$ & $1.28(0.81-2.02)$ \\
\hline \multicolumn{5}{|c|}{ Peripheral oxygen saturation (\%) } \\
\hline $96-100^{*}$ & 1.00 & & & \\
\hline $91-95$ & $1.16(1.06-1.26)$ & $1.15(1.00-1.32)$ & $1.16(1.02-1.32)$ & $1.04(0.85-1.26)$ \\
\hline $86-90$ & $1.39(1.18-1.65)$ & $1.35(1.00-1.84)$ & $1.41(1.10-1.82)$ & $1.44(1.01-2.05)$ \\
\hline $81-85$ & $2.43(1.90-3.10)$ & $3.72(2.48-5.60)$ & $2.18(1.49-3.19)$ & $1.67(0.97-2.90)$ \\
\hline $0-80$ & $3.00(2.41-3.74)$ & $3.34(2.33-4.81)$ & $3.55(2.55-4.95)$ & $1.92(1.11-3.32)$ \\
\hline \multicolumn{5}{|c|}{ Respiratory rate (/min) } \\
\hline Not registered* & 1.00 & & & \\
\hline $0-9$ & $4.15(2.96-5.82)$ & $3.71(2.37-5.81)$ & $4.29(2.38-7.74)$ & $6.59(2.02-21.50)$ \\
\hline $10-19$ & $2.37(2.01-2.79)$ & $2.28(1.82-2.87)$ & $2.27(1.73-2.97)$ & $2.58(1.58-4.19)$ \\
\hline $20-29$ & $3.15(2.66-3.73)$ & $3.11(2.45-3.96)$ & $2.96(2.25-3.90)$ & $3.53(2.16-5.76)$ \\
\hline$>30$ & $5.16(4.19-6.35)$ & $5.59(4.07-7.69)$ & $5.61(4.03-7.81)$ & $5.20(3.00-9.03)$ \\
\hline \multicolumn{5}{|c|}{ Heart rate (beats/min) } \\
\hline$>125$ & $2.53(2.23-2.87)$ & $2.69(2.23-3.24)$ & $2.10(1.71-2.59)$ & $2.92(2.11-4.02)$ \\
\hline $101-125$ & $1.52(1.36-1.68)$ & $1.45(1.24-1.69)$ & $1.55(1.31-1.83)$ & $1.37(1.03-1.81)$ \\
\hline $76-100$ & $1.10(1.01-1.19)$ & $1.00(0.88-1.13)$ & $1.20(1.05-1.37)$ & $1.18(0.96-1.46)$ \\
\hline $51-75^{*}$ & 1.00 & & & \\
\hline $0-50$ & $1.34(1.08-1.65)$ & $1.10(0.74-1.62)$ & $1.25(0.90-1.74)$ & $2.11(1.38-3.23)$ \\
\hline \multicolumn{5}{|l|}{ Temperature $\left({ }^{\circ} \mathrm{C}\right)$} \\
\hline Not registered* & 1.00 & & & \\
\hline$\geq 40$ & $0.50(0.32-0.78)$ & $0.53(0.29-0.97)$ & $0.53(0.25-1.14)$ & $0.30(0.07-1.28)$ \\
\hline $38-39$ & $0.35(0.30-0.41)$ & $0.38(0.30-0.49)$ & $0.37(0.28-0.47)$ & $0.28(0.18-0.43)$ \\
\hline $35-37$ & $0.64(0.59-0.70)$ & $0.63(0.56-0.71)$ & $0.66(0.58-0.76)$ & $0.64(0.51-0.79)$ \\
\hline $31-34$ & $1.36(1.00-1.84)$ & $1.58(1.02-2.43)$ & $1.14(0.67-1.92)$ & $1.41(0.62-3.17)$ \\
\hline $0-30$ & $3.95(1.59-9.82)$ & $5.90(1.36-25.66)$ & $1.89(0.36-9.95)$ & $11.6(1.64-81.7)$ \\
\hline
\end{tabular}

The following potential confounders were entered in the model for through backward stepwise regression: age, gender, top 10 chief complaints, hospital, treating specialty, $\mathrm{SpO}_{2}$ temperature, proxies of disease severity (triage level, fluid administration, Glasgow Coma Scale, blood gas analyses) and proxies of disease comorbidities/complexity (number of consultations, lab tests, performed radiological tests).

* Reference category in the regression analyses.

AORs, adjusted odds ratios; ICU, intensive care unit; MCU, medium care unit.

population corresponds with previous studies in traumatic brain injury, sepsis, hypertension and unselected ED patients. ${ }^{9} 2021$ Our study suggests that a single cut-off is also absent for $\mathrm{SpO}_{2}$ correspondent to earlier findings in a study by Ljunggren et al in 96512 unselected ED patients. ${ }^{22}$ Interestingly, temperatures above $35.5^{\circ} \mathrm{C}$, that is, normal temperature and fever, were not significantly associated with mortality in our and Ljunggren's study. This finding is in contrast to what has been suggested in several risk stratification tools, like the national and modified
Early Warning Score (EWS), in which points are assigned for high temperature. ${ }^{23}$ It should be noted, however, that the original EWS was not intended to be a predictor of outcome but rather to be a simple tool to assist inexperienced nursing or medical staff in recognising patients at risk for deterioration and in securing immediate, more experienced help. ${ }^{24}$

In addition to a threshold for temperature, we also found a single cut-off for MAP and RR. RR was the least registered vital sign, but our findings confirm the importance of RR given the 
strong association with mortality and the higher odds for ICU/ MCU admission if RR was registered, compared with if it was not registered. The threshold we found for MAP $<70 \mathrm{~mm} \mathrm{Hg}$ was similar to the thresholds used in several guidelines. ${ }^{13}$ Interestingly, the odds for ICU admission only started to increase with MAP below $60 \mathrm{~mm} \mathrm{Hg}$. Probably, physicians are less triggered to admit patients to an ICU or MCU with a MAP between 60 and $80 \mathrm{~mm} \mathrm{Hg}$. This discrepancy between the associations of MAP and in-hospital mortality versus ICU admission warrants further research as it may reflect unrecognised hypotension, and patients with higher MAPs may need ICU admission.

Different from other studies, we have studied the effect of age on associations between vital signs and outcome. Older patients had larger increases in the absolute risk for mortality with changing vital sign categories compared with younger patients, while the relative risk for mortality had similar increases. Previously, the use of different single cut-off values for SBP in trauma patients have been suggested for advanced age. ${ }^{25}$ Although prognosis has been found to deteriorate at higher SBP threshold, our data suggest not to use a single cut-off at all.

The findings of the present study have several implications for clinical practice. Although a single cut-off may be convenient and easy to use, our first finding suggests that the use of a cut-off for several vital signs in risk stratification tools and acute care guidelines are inappropriate. ${ }^{1-6}$ The use of a single cut-off suggests that above or below this specific threshold, prognosis changes substantially. Using only a single threshold, like for SBP or RR in the qSOFA score, does not mean that the chosen threshold for SBP of $100 \mathrm{~mm} \mathrm{Hg}$ discriminates better between low-risk and high-risk patients than a threshold of 90,110, 120, 130, 140 $\mathrm{mm} \mathrm{Hg}$ and so on, as has been demonstrated previously for SBP in traumatic brain injury. ${ }^{9}$ Even if a single cut-off exists, that is, if an association between outcome and a vital sign is only apparent from a certain value, dichotomous risk stratification would ignore further increase of the risk with more extreme values of the vital sign. This implicates that several risk stratification tools and acute care guidelines could be improved by using numerical scores based on AORs or predicted mortality rather than using dichotomous variables. ${ }^{1-6}$ Currently, these grading scores are already used in EWS, although the scores in the EWS are not supported by AORs derived from prediction models. ${ }^{23}$

APACHE II is a good example of a numerical score for ICU patients. ${ }^{26}$ Similar risk stratification scores may be developed for the ED to improve recognition, initial resuscitation and disposition.

Our second main finding implicates that risks scores need to be age-adjusted. The effect of vital signs on mortality did change with advancing age, because baseline risk for older age was higher. For example, if SBP decreased and the risk on in-hospital mortality increased fourfold, the effect on younger patients with a baseline mortality risk, that is, $0.5 \%$ would be much smaller than the effect on older patients with a baseline mortality risk, that is, $4.0 \%$. More points may be assigned in a numerical score for older age for similar vital signs.

Interestingly, the subgroup analyses in sepsis and trauma patients suggests that the aetiology of changes in vital signs does not affect the impact of vital signs on relevant outcomes. Future studies should incorporate our findings in development of risk stratification tools and acute care guidelines for ED patients using numerical scores based on statistical risks.

Our study has several strengths like a large sample size with unselected patients and the multicentre design. Several limitations merit emphasis. First, this observational study used the NEED, which lacks information about the medical history and comorbidities of patients. However, to overcome this, we used variables that are associated with comorbidities and complexity. ${ }^{16}$ Second, information bias cannot be completely ruled out although data collection was largely automatised and only reliably registered variables are collected in the NEED. Finally, we missed outcomes of patients who were hospitalised elsewhere. However, the sensitivity analyses in which we considered these patients as deceased, yielded similar results.

\section{CONCLUSION}

The use of a single cut-off for each vital sign in acute care guidelines and risk stratification tools deserves scrutiny, as our results show that clinical outcomes increase gradually or in a U-shaped fashion with changing vital signs. A threshold for MAP, RR and temperature exists; however, using these single cut-offs would ignore further increase of risk with more extreme values of these vital signs. Age-adjusted numerical scores will improve risk stratification, as older patients have larger absolute increases in mortality with changing vital signs, even after adjusting for confounders.

\section{Author affiliations}

${ }^{1}$ Emergency Department, Maxima Medical Centre, Veldhoven, Noord-Brabant, The Netherlands

${ }^{2}$ Emergency Department, Leiden University Medical Centre, Leiden, Zuid-Holland, The Netherlands

${ }^{3}$ Emergency Department, Admiraal De Ruyter Hospital, Goes, Zeeland, The Netherlands

${ }^{4}$ Emergency Department, University Medical Centre Groningen, Groningen, The Netherlands

${ }^{5}$ Emergency Department, Radboud University Medical Centre, Nijmegen, The Netherlands

${ }^{6}$ Emergency Department, Medical Centre Leeuwarden, Leeuwarden, Friesland, The Netherlands

${ }^{7}$ Emergency Department, Elisabeth-TweeSteden Hospital, Tilburg, Noord-Brabant, The Netherlands

${ }^{8}$ Emergency Department, Medical Centre Haaglanden, Den Haag, Zuid-Holland, The Netherlands

${ }^{9}$ Department of Biostatistics, Leiden Universitair Medical Centre, Leiden, ZuidHolland, The Netherlands

${ }^{10}$ Intensive Care, Leiden University Medical Centre, Leiden, Zuid-Holland, The Netherlands

\section{Twitter Ewoud ter Avest @ewoudteravest}

Contributors BdG devised and designed the study, collected data, contributed to the analyses and edited the manuscript. BC and RD collected and analysed data and wrote the manuscript. MIG, EtA, OS, HL, RH and RR are founders of the NEED, collected data and edited the manuscript. EvZ helped with statistical analyses and edited the manuscript. EdJ edited the manuscript. BdG takes full responsibility for the study and acts as a guarantor. All authors have read and approved the manuscript.

Funding $B C$ and $B d G$ received financial funding from the Dutch Emergency Medicine Research Fund (SGO-fonds, award number NA).

Competing interests None declared.

Patient consent for publication Not applicable.

Ethics approval The study was approved by the medical ethics committee of the LUMC who waived the need for individual consent because of the purely observational character of the study. The study was registered at the Netherlands Trial Register (ID NL8422).

Provenance and peer review Not commissioned; externally peer reviewed.

Data availability statement Data are available on reasonable request. Deidentified participant data are available on reasonable request from the corresponding author.

Supplemental material This content has been supplied by the author(s). It has not been vetted by BMJ Publishing Group Limited (BMJ) and may not have been peer-reviewed. Any opinions or recommendations discussed are solely those of the author(s) and are not endorsed by BMJ. BMJ disclaims all liability and responsibility arising from any reliance placed on the content. Where the content includes any translated material, BMJ does not warrant the accuracy and reliability of the translations (including but not limited to local regulations, clinical guidelines, 
terminology, drug names and drug dosages), and is not responsible for any error and/or omissions arising from translation and adaptation or otherwise.

Open access This is an open access article distributed in accordance with the Creative Commons Attribution Non Commercial (CC BY-NC 4.0) license, which permits others to distribute, remix, adapt, build upon this work non-commercially, and license their derivative works on different terms, provided the original work is properly cited, appropriate credit is given, any changes made indicated, and the use is non-commercial. See: http://creativecommons.org/licenses/by-nc/4.0/.

\section{ORCID iDs}

Bart GJ Candel http://orcid.org/0000-0002-4500-9960

Ewoud ter Avest http://orcid.org/0000-0002-1462-6130

Heleen Lameijer http://orcid.org/0000-0001-9378-7743

\section{REFERENCES}

1 Rhodes A, Evans LE, Alhazzani W, et al. Surviving sepsis campaign: international guidelines for management of sepsis and septic shock: 2016. Intensive Care Med 2017:43:304-77.

2 Metlay JP, Waterer GW, Long AC, et al. Diagnosis and treatment of adults with community-acquired pneumonia. An official clinical practice guideline of the American thoracic Society and infectious diseases Society of America. Am J Respir Crit Care Med 2019;200:e45-67.

3 American College of S, Committee on T. ATLS : advanced trauma life support for doctors : student course manual. Chicago, IL: American College of Surgeons, 2008.

4 Adams HP, del Zoppo G, Alberts MJ, et al. Guidelines for the early management of adults with ischemic stroke: a guideline from the American heart Association/ American stroke association stroke Council, clinical cardiology Council, cardiovascular radiology and intervention Council, and the atherosclerotic peripheral vascular disease and quality of care outcomes in research interdisciplinary working groups: the American Academy of Neurology affirms the value of this guideline as an educational tool for neurologists. Stroke 2007;38:1655-711.

5 Bone RC. Immunologic dissonance: a continuing evolution in our understanding of the systemic inflammatory response syndrome (SIRS) and the multiple organ dysfunction syndrome (MODS). Ann Intern Med 1996;125:680-7.

6 Champion HR, Sacco WJ, Copes WS, et al. A revision of the trauma score. J Trauma 1989;29:623-9.

7 Shapiro NI, Wolfe RE, Moore RB, et al. Mortality in emergency department sepsis (MEDS) score: a prospectively derived and validated clinical prediction rule. Crit Care Med 2003;31:670-5.

8 Seymour CW, Liu VX, Iwashyna TJ, et al. Assessment of clinical criteria for sepsis: for the third International consensus definitions for sepsis and septic shock (Sepsis-3). JAMA 2016:315:762-74.

9 Spaite DW, Hu C, Bobrow BJ, et al. Mortality and prehospital blood pressure in patients with major traumatic brain injury: implications for the hypotension threshold. JAMA Surg 2017;152:360-8.
10 Warmerdam M, Baris L, van Liebergen M, et al. The association between systolic blood pressure and in-hospital mortality in older emergency department patients who are hospitalised with a suspected infection. Emerg Med J 2018;35:619-22.

11 de Groot B, Stolwijk F, Warmerdam M, et al. The most commonly used disease severity scores are inappropriate for risk stratification of older emergency department sepsis patients: an observational multi-centre study. Scand I Trauma Resusc Emerg Med 2017; 25:91.

12 Pirneskoski J, Lääperi M, Kuisma M, et al. Ability of prehospital news to predict 1-day and 7-day mortality is reduced in older adult patients. Emerg Med J 2021;38:913-8.

13 Chester JG, Rudolph JL. Vital signs in older patients: age-related changes. J Am Med Dir Assoc 2011;12:337-43.

14 Cretikos MA, Bellomo R, Hillman K, et al. Respiratory rate: the neglected vital sign. Med J Aust 2008;188:657-9.

15 Vittinghoff E, McCulloch CE. Relaxing the rule of ten events per variable in logistic and COX regression. Am J Epidemiol 2007;165:710-8.

16 van der Veen D, Heringhaus C, de Groot B. Appropriateness, reasons and independent predictors of consultations in the emergency department (ED) of a Dutch tertiary care center: a prospective cohort study. PLoS One 2016;11:e0149079.

17 Woods RA, Lee R, Ospina MB, et al. Consultation outcomes in the emergency department: exploring rates and complexity. CJEM 2008;10:25-31.

18 Rubulotta F, Marshall JC, Ramsay G, et al. Predisposition, insult/infection, response, and organ dysfunction: a new model for staging severe sepsis. Crit Care Med 2009;37:1329-35.

19 Lim WS, van der Eerden MM, Laing R, et al. Defining community acquired pneumonia severity on presentation to hospital: an international derivation and validation study. Thorax 2003; 58:377-82.

20 Klang E, Soffer S, Shimon Shahar M, et al. Association of normal systolic blood pressure in the emergency department with higher in-hospital mortality among hypertensive patients. J Clin Hypertens 2019:21:1841-8.

21 Kristensen AKB, Holler JG, Mikkelsen S, et al. Systolic blood pressure and short-term mortality in the emergency department and prehospital setting: a hospital-based cohort study. Crit Care 2015;19:158.

22 Ljunggren M, Castrén M, Nordberg M, et al. The association between vital signs and mortality in a retrospective cohort study of an unselected emergency department population. Scand I Trauma Resusc Emerg Med 2016;24:21.

23 Alam N, Hobbelink EL, van Tienhoven AJ, et al. The impact of the use of the early warning score (EWS) on patient outcomes: a systematic review. Resuscitation 2014;85:587-94.

24 Morgan RJM, Wright MM. In defence of early warning scores. Br J Anaesth 2007:99:747-8.

25 Oyetunji TA, Chang DC, Crompton JG, et al. Redefining hypotension in the elderly: normotension is not reassuring. Arch Surg 2011;146:865-9.

26 Knaus WA, Draper EA, Wagner DP, et al. Apache II: a severity of disease classification system. Crit Care Med 1985;13:818-29. 